\title{
Efficient carrier-envelope phase tunable mid-infrared frequency combs based on CW-seeded optical parametric generation
}

\author{
Mikhail Roiz ${ }^{1, *}, J u i-Y u$ Lai $^{2}$, Juho Karhu ${ }^{3}$ and Markku Vainio ${ }^{1,4}$ \\ ${ }^{1}$ Department of Chemistry, University of Helsinki, FI-00560, Helsinki, Finland \\ ${ }^{2}$ HC Photonics Corp. Hsinchu Science Park, Hsinchu 30078, Taiwan \\ ${ }^{3}$ Metrology Research Institute, Aalto University, Espoo, FI-00076, Finland \\ ${ }^{4}$ Photonics Laboratory, Physics Unit, Tampere University, Tampere, FI-33101, Finland
}

\begin{abstract}
We present an efficient method for generating frequency combs in the mid-infrared (MIR) spectral range in bulk lithium niobate crystals as well as in waveguides. Our approach is simple and robust, since it is based on single-pass configuration of femtosecond Optical Parametric Generation (OPG) seeded by a telecom continuous-wave laser. Precise and fast tuning of the seed laser allows to rapidly change the offset frequency of the generated MIR comb independent of its repetition rate. The MIR comb offset frequency is inherently known, so its direct detection is not required. An additional degree of freedom that further complements the versatility of our light source is a unique way of repetition rate or mode spacing adjustments. This is achieved by our new concept of coherent multi-seeding that depending on the configuration allows for pulse train modulation and mode spacing, or repetition rate division by an integer number.
\end{abstract}

\section{Introduction}

Mid-infrared (MIR) Optical Frequency Comb (OFC) technology is a rapidly evolving scientific field since it has many crucial applications including frequency metrology, high-harmonic generation, microscopy and high-resolution spectroscopy. Especially in highresolution spectroscopy, high-quality MIR OFCs are of great importance because they allow for parallel detection of tens of thousands absorption lines simultaneously with metrological precision [1]. Direct generation of MIR OFCs in Mode-Locked Lasers (MLL) is challenging, thus requiring parametric down-conversion techniques to be involved. However, the most common ways for MIR OFC generation based on parametric down-conversion namely Optical Parametric Oscillation [2] and Difference Frequency Generation [3] - are technically quite demanding and complicated. In this conference contribution, we demonstrate an alternative way to generate MIR OFCs based on Optical Parametric Generation (OPG), which is remarkably simple and highly versatile.

\section{Experimental setup}

Our approach is general and can be used in any nondegenerate OPG system, however we have implemented the method using a readily available and popular nonlinear material - $\mathrm{MgO}$ doped periodically poled lithium niobate (MgO:PPLN). Here we demonstrate two $\mathrm{CW}$-seeded OPG implementations - one is based on bulk crystals and the other on nonlinear waveguide technology in MgO:PPLN. The crystal/waveguide is pumped by a near-infrared MLL (250 MHz repetition rate, $100 \mathrm{fs}$ pulse duration) centred at $1 \mu \mathrm{m}$ wavelength, as schematically shown in Fig. 1 (a). The seeding is done in the signal region, which coincides with the convenient telecom

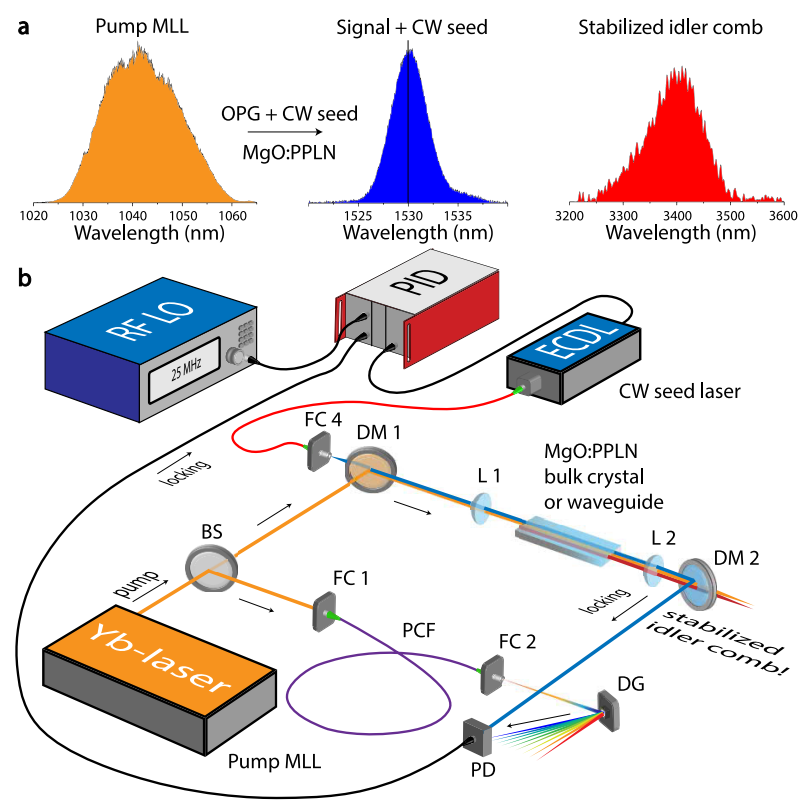

Fig. 1. (a) CW-seeded OPG concept (spectra are shown in the linear scale with arbitrary units for the case of bulk crystal). (b) Experimental setup. BS: beam splitter, FC: fiber collimator, DG: diffraction grating, DM: dichroic mirror, L: lens, PD: photodiode, PID: Proportional-Integral-Derivative controller, ECDL: External Cavity Diode Laser, PCF: Photonic Crystal Fiber.

\footnotetext{
* Corresponding author: mikhail.roiz@helsinki.fi
} 
wavelengths. The idler comb typically has an instantaneous spectral coverage of $100 \mathrm{~nm}$ (full-width-athalf-maximum) for the bulk system and over $300 \mathrm{~nm}$ for the waveguides. The bulk system is realized using fanout crystal structure making the idler comb easily tunable across $3325-4000 \mathrm{~nm}\left(2380-3030 \mathrm{~cm}^{-1}\right)$. For more details on the bulk system as well as on waveguide see [4] and [5], respectively.

In this work we show that the $\mathrm{CW}$ seed laser can be phase-locked to a supercontinuum generated by the pump MLL, which guarantees full stabilization of the MIR comb $[4,5]$. When the signal comb of the OPG is seeded by a such phase-locked CW seed laser (see Fig. 1 (b)), it means that the signal comb shares the same CarrierEnvelope Offset (CEO) as the pump MLL, but with an additional frequency offset. This additional frequency offset is precisely defined by a conventional radio frequency (RF) local oscillator (RF LO in Fig. 1 (b)) used in the phase-locking process. Thus, by adjusting the RF LO frequency, one can continuously tune the signal and, most importantly, the idler CEO.

Table 1. Comparison of bulk and waveguide systems.

\begin{tabular}{|c|c|c|}
\hline Parameter & Bulk & Waveguide \\
\hline Threshold & $8-16 \mathrm{~nJ}$ & $25 \mathrm{pJ}$ \\
\hline Max. idler average power & $700 \mathrm{~mW}$ & $5.8 \mathrm{~mW}$ \\
\hline Max. conversion efficiency & $40 \%$ & $74 \%$ \\
\hline Pump-to-idler conversion & $13 \%$ & $23 \%$ \\
\hline Instantaneous bandwidth & $100 \mathrm{~nm}$ & $>300 \mathrm{~nm}$ \\
\hline Min. integrated RIN & $0.018 \%$ & $<0.0047 \%$ \\
\hline
\end{tabular}

\section{Results}

In Table 1 one can see a comparison of the main parameters of $\mathrm{CW}$-seeded OPG in the bulk and waveguide systems. It is evident that the bulk system can be used for high power applications since it has a lot of output power: $700 \mathrm{~mW}$ of average power at $250 \mathrm{MHz}$ repetition rate. However, the waveguide system has much lower threshold of just $25 \mathrm{pJ}$ and a larger instantaneous optical bandwidth of over $300 \mathrm{~nm}$ (see Fig. 2 (a)). Additionally, in waveguides, we have achieved a remarkably high conversion efficiency of $74 \%$, representing state-of-theart for OPG systems [6].

Another important figure of merit is the Relative Intensity Noise (RIN). The importance of RIN in spectroscopy applications is hard to overestimate, since it directly defines detection limit or signal-to-noise ratio of the measurement. We show that in the OPG process RIN can be substantially reduced by $\mathrm{CW}$ seeding, which is evident from Fig. 2 (b). Remarkably, the integrated RIN in waveguides is much lower than in bulk systems because in waveguides one does not need to use highpower amplifiers (that induce additional intensity noise) for the pump laser. Consequently, the root-mean-square RIN of the MIR comb generated in a waveguide is smaller than $5 \times 10^{-5}$ when integrated from $10 \mathrm{~Hz}$ to $2 \mathrm{MHz}$, where the noise is mostly below the detection limit.

Next we demonstrate how well the CEO of the two idler combs can be stabilized. We compare the two combs to one another using the same pump laser but with two different $\mathrm{CW}$ seed lasers locked to the supercontinuum as shown in Fig. 1 (b). The relative offset beat note can be seen in Fig. 2 (c) with sub-Hz linewidth, which is limited by the RF spectrum analyser. By performing frequency counting measurement of this beat note, we can conclude that the relative stability of the two combs is excellent with just $36 \mathrm{mHz}$ standard deviation around the target frequency. The noise in this measurement averages out as white noise confirming that there are no relative drifts between the combs.

We would like to note that the bulk and waveguide implementations have excellent versatility in terms of offset frequency adjustments. The offset can be freely tuned, scanned or modulated with metrological precision $[4,5]$. Our scheme does not have any cavities meaning that the signal/idler comb repetition rate can be freely adjusted by simply changing the pump laser repetition rate. Moreover, there is another useful degree of freedom that makes our light source even more versatile and flexible. Here we introduce a new concept of coherent $\mathrm{CW}$ multi-seeding that allows one to divide the repetition rate of the generated signal and idler combs by an integer fraction.
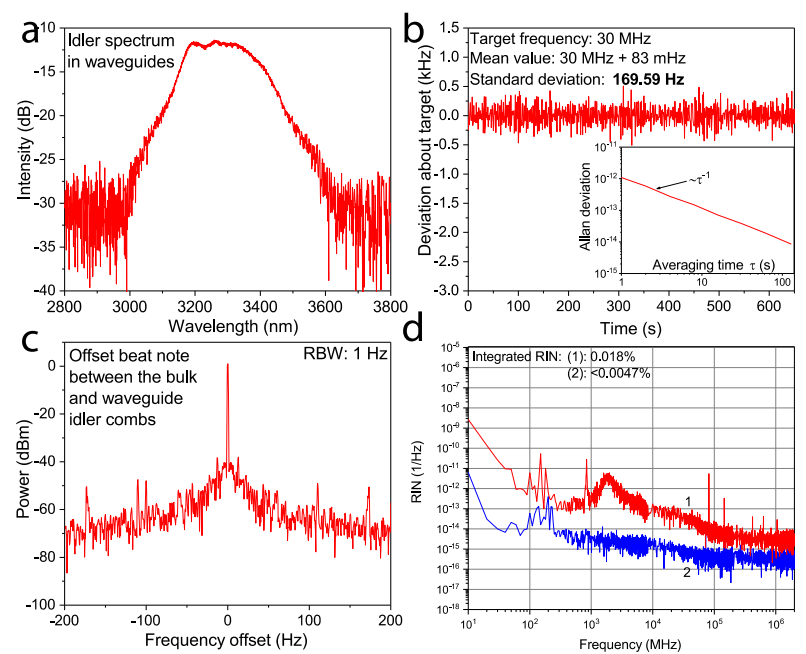

Fig. 2. (a) Typical idler spectrum in waveguides; (b) Relative Intensity Noise (RIN): (1) saturated OPG in bulk crystal with CW seeding; (2) same as (1) but in waveguides; (c) Offset beat note between the bulk and waveguide idler combs (pump laser is the same for both combs, see [5] for details); (d) Frequency counting experiment of the offset beat note in (c).

The term coherent CW multi-seeding refers to seeding of the OPG process with more than one mutually phase coherent $\mathrm{CW}$ lasers. In the simplest form of multi-seeding - namely dual-seeding - this approach requires two single mode CW lasers that are phase-locked to one another. The relative optical phases and frequencies of the seed lasers can be controlled by two independent RF LOs involved in the phase locking procedure. If we set the optical frequency difference to be exactly half of the pump laser repetition rate $(250 / 2 \mathrm{MHz}$ in our case), then the $C W$ seeded OPG process will start up for both seed lasers simultaneously. It leads to the generation 

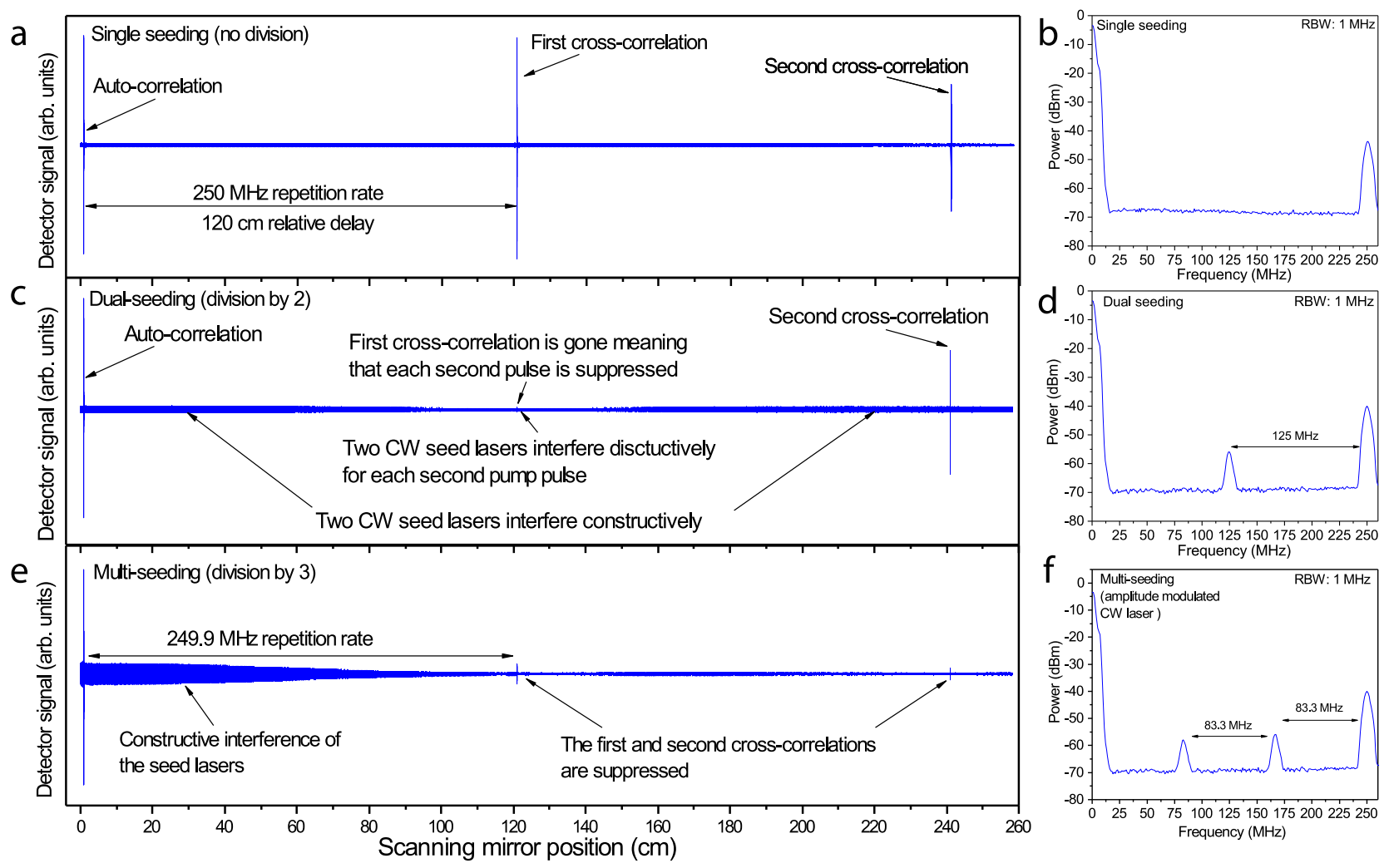

Fig. 3. (a) Interferogram in the case of single CW-seeded OPG; (b) RF spectrum of the signal/idler repetition rate for (a): (c) Interferogram for dual $\mathrm{CW}$-seeded OPG corresponding to repetition rate division by a factor of 2; (d) RF spectrum of the signal/idler repetition rate for (c); (e) Interferogram for multi (one $\mathrm{CW}$ laser is amplitude modulated) $\mathrm{CW}$-seeded OPG corresponding to repetition rate division by a factor of 3; (f) RF spectrum of the signal/idler repetition rate for (e).

of two signal/idler frequency combs within one laser pulse, optical frequencies of which are separated by exactly the frequency separation between the CW seed lasers. The dual-seeding configuration divides the repetition rate of the signal and idler combs in both frequency and time domain only when the $C W$ seed lasers are separated exactly by half of the pump repetition rate. If the two seed lasers are separated by $83.3 \mathrm{MHz}(250 / 3$ $\mathrm{MHz}$ ) pulse train modulation takes place (instead of pulse picking), where one of the pulses can be removed from the pulse train. This effect can be seen in the oscilloscope traces of Fig. 4, where we also exemplify the case of repetition rate division by a factor of 4 . In order to efficiently divide the repetition rate in time domain one needs more than two seed lasers. Luckily, it can be easily done by using one CW laser with amplitude modulation. When a CW laser is amplitude modulated at the frequency of $83.3 \mathrm{MHz}$ (division by 3 ), it generates additional sidebands (18 in our case) separated by $83.3 \mathrm{MHz}$ serving as multi-seeding source. In Fig. 3e one can see how both the first and second cross-correlations are suppressed on the interferogram meaning that each second and third pulse is removed from the pulse train. In the frequency domain repetition rates of signal and idler combs are divided by 3 exactly the same way as it was done using dual-seeding approach (Fig. 3f).

Extreme repetition rate division - namely pulse picking at low frequencies - can be obtained by using a single seed laser, which is passed through a fast intensity modulator driven by short electrical pulses. The modulator can be used to switch the seed pulses on or off, hence gating the OPG process. This makes it possible to produce a repetition rate different from that of the pump (which is fixed at $250 \mathrm{MHz}$ in all experiments), leading to repetition rate division by any integer. Alternatively, the seed laser pulses can be configured for arbitrary on/off modulation of the femtosecond pulses generated by the OPG process.

To summarize, in our conference contribution we demonstrate a simple concept for MIR frequency comb generation and provide its rigorous characterization. Our system makes it easy to stabilize and dynamically control the MIR comb offset frequency along with the free tuning of the repetition rate. Additionally, there is no need to directly detect the MIR comb offset frequency because its value is inherently known. We believe that the presented method is promising for a variety of applications in spectroscopy such as dual-comb spectroscopy, combassisted and Fourier-transform spectroscopy as well as in other scientific fields including microscopy and highharmonic generation. Furthermore, our results in waveguides open up opportunities for efficient downconversion of high repetition rate frequency combs $(>1$ $\mathrm{GHz}$ ), which is important for fast spectroscopic measurements, for example in reaction kinetics. This MIR comb generation method has an additional important advantage - one can perform repetition rate division directly in the OPG process using coherent multi-seeding approach. It helps to further improve the resolution of spectroscopic measurements by increasing the number of optical modes in the MIR comb (making it denser in frequency domain). In the pulse picking configuration one

\footnotetext{
* Corresponding author: mikhail.roiz@,helsinki.fi
} 
can selectively start the OPG process for any given pump pulse. Effectively, this filters out the pulse train and picks only those signal and idler pulses that are desired. This particular scheme can be applied in optical parametric chirped pulse amplifiers as a seed source since the seed sources need to have low repetition rate $(<200 \mathrm{kHz})$ [8].

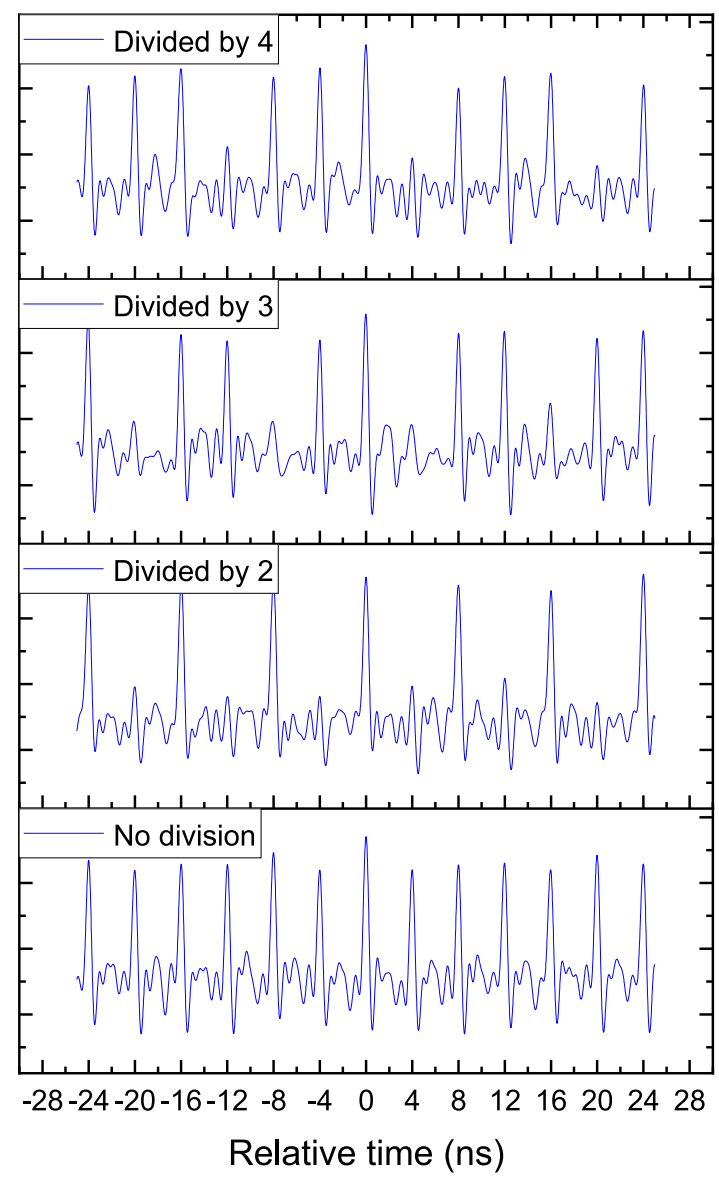

Fig. 4. Oscilloscope traces of the signal (same for idler) pulse train in different repetition rate division configurations in the dual-seeded case.

\section{References}

1. 1. A. V. Muraviev, V. O. Smolski, Z. E. Loparo, K. L. Vodopyanov, Nat. Photonics 12, 209 (2018).

2. M. Vainio and L. Halonen, Phys. Chem. Chem. Phys. 18, 4266 (2016).

3. G. Soboń, T. Martynkien, P. Mergo, L. Rutkowski, A. Foltynowicz, Opt. Lett. 42, 1748 (2017).

4. M. Roiz, K. Kumar, J. Karhu, M. Vainio, APL Photonics 6, 026103 (2021).

5. M. Roiz, J. Lai, J. Karhu, M. Vainio, Opt. Lett. 46, 4037-4040 (2021).

6. M. Jankowski, N. Jornod, C. Langrock, B. Desiatov, A. Marandi, M. Lončar, M. M. Fejer, arXiv preprint arXiv:2104.07928 (2021).

7. J. Fan, W. Chen, C. Gu, Y. Song, L. Chai, C. Wang, M. Hu, Opt. Express 25, 24594 (2017).

8. U. Elu, M. Baudisch, H. Pires, F. Tani, M. H. Frosz, F. Köttig, A. Ermolov, P. St.J. Russell, J. Biegert, Optica 4, 1024-1029 (2017). 